\title{
A Convolution-Free Mixed Finite-Element Time-Domain Method for General Nonlinear Dispersive Media
}

\author{
David S. Abraham, Student Member, IEEE, and Dennis D. Giannacopoulos, Senior Member, IEEE
}

\begin{abstract}
In this paper, a mixed Finite-Element Time-Domain (FETD) method is presented for the simulation of electrically complex materials, including general combinations of linear dispersion, instantaneous nonlinearity, and dispersive nonlinearity. Using both edge and face elements, the presented method offers greater geometric flexibility than existing Finite-Difference TimeDomain (FDTD) implementations, and in contrast to existing nonlinear FETD methods, also incorporates both linear and nonlinear material dispersion. Dielectric nonlinearity is incorporated into the Crank-Nicolson mixed FETD formulation via a straightforward Newton-Raphson approach, for which the associated Jacobian is derived. Moreover, the dispersion is modeled via the Möbius z-transform method, yielding a simpler more general algorithm. The method's accuracy and convergence are verified, and its capability demonstrated via the simulation of several nonlinear phenomena, including temporal and spatial solitons in two spatial dimensions.
\end{abstract}

Index Terms-Nonlinear Media; Dispersive Media; FiniteElement Methods; Time-Domain Analysis.

\section{INTRODUCTION}

$\mathbf{T}$ HE need for fast, efficient, and effective telecommunications has become paramount in today's world as people and devices are ever more connected. Consequently, the requirement for rapid and reliable communications infrastructure has also grown tremendously, resulting in the research and development of numerous electromagnetic devices for the transmission and reception of data. Most notably, the field of nonlinear optics has yielded significant advancements, and continues to be an important evolving area of research, propelled by additional advances in fabrication and material sciences.

With the ubiquity of these devices, the need for design tools and simulation techniques, as low-cost alternatives to expensive prototyping and physical experimentation, has also increased. As a result, a myriad of methods and techniques have been developed to aid in the study and design of electromagnetic devices exhibiting complex material behaviour,

Manuscript received fany 5, 2018; revised 2018; accepted January 5, 2018. Date of publication January 5, 2018; date of current version August 8, 2018. This work was supported by the Natural Sciences and Engineering Research Council (NSERC) of Canada.

The authors are with the Department of Electrical and Computer Engineering, McGill University, Montréal, Québec, Canada H3A 0E9 (e-mail: david.abraham@mail.mcgill.ca; dennis.giannacopoulos@mcgill.ca)

Color versions of one or more of the figures in this paper are available online at http://ieeexplore.ieee.org.

Digital Object Identifier \#\#\#\#\#\#\# such as material dispersion and dielectric nonlinearity. Such methods could have wide ranging applications, even outside the world of optics. For example, it has been known for some time that biological tissues can exhibit both dispersive [1] and nonlinear [2] behaviours. Such techniques could thus even be used to study abnormalities in biological systems, such as metabolic disorders within mitochondria [3].

Many of these methods, however, tend to include simplifying assumptions about the nature of the propagating waves, which restricts their applicability and versatility. Furthermore, while full-wave time-domain methods, in which no simplifications have been made, have been developed, the current existing families of methods either have geometric constraints, do not yet have integration for more general combinations of both dielectric nonlinearity and dispersion, or do not easily scale to arbitrary dispersive and nonlinear orders.

In this paper, a novel technique based upon the mixed FiniteElement Time-Domain (FETD) method will be presented to address these shortcomings. In contrast to existing techniques, the presented method can handle general arbitrary combinations of material dispersion and nonlinearity. Moreover, by leveraging both the implicit mixed FETD method and ztransform techniques, the presented scheme has geometric flexibility, stability, and scales easily to higher order phenomena. In the following sections, a brief summary of existing approximative and full-wave techniques will be discussed, followed by an overview of the standard mixed FETD method. The new method will then be derived, and its accuracy and convergence verified. Lastly, it will also be used to demonstrate the occurrence of several well-known nonlinear phenomena.

\section{BACKGROUND}

\section{A. Nonlinear Theory}

Electromagnetic devices exhibiting material dispersion, dielectric nonlinearity, or both, have been in use for many years and have resulted in a variety of techniques for their design and simulation. The underlying theory remains Maxwell's equations; however the associated material permittivity is altered to reflect the complex behaviour. Specifically, in the case of material dispersion, convolutions are introduced in the time-domain, as the permittivity is now frequency dependent. In the nonlinear case, the permittivity is dependent on the 
magnitude of the electric field. This is often expressed in terms of the material's polarization, $\vec{P}$, namely:

$$
\vec{D}=\epsilon_{0} \vec{E}+\vec{P}(t, E)
$$

in which $\epsilon_{0}$ is the permittivity of free space, $\vec{D}$ is the electric flux density, $\vec{E}$ is the electric field, $E=|\vec{E}|$, and convolution occurs within $\vec{P}$. Furthermore, it is often convenient to expand the material polarization as a power series in $\vec{E}$. Such an expansion would in general involve tensors [4], however for many material models an adequate expansion is:

$$
\vec{P}=\epsilon_{0} \chi^{(1)}(t) * \vec{E}+\epsilon_{0} \chi^{(3)}\left(\alpha E^{2}+(1-\alpha) g(t) * E^{2}\right) \vec{E}
$$

in which the susceptibility $\chi^{(1)}$ models linear dispersion, the susceptibility $\chi^{(3)}$ an instantaneous Kerr and/or dispersive stimulated Raman nonlinearity (with the $\alpha$ term controlling their relative strengths) [5], [6], and $*$ denoting convolution. The $\chi^{(2)}$ term has been omitted, since it is usually absent due to inversion symmetry [4]. Nonetheless, the method to follow can easily incorporate such effects if desired. This $\vec{D}$ field expression can then be inserted into Maxwell's equations, yielding a nonlinear wave equation of the form [4]:

$$
\nabla^{2} \vec{E}-\mu_{0} \epsilon_{L} * \frac{\partial^{2} \vec{E}}{\partial t^{2}}=\mu_{0} \frac{\partial^{2} \vec{P}_{N L}}{\partial t^{2}}
$$

where $\epsilon_{L}$ is the usual linear dispersive term (related to $\chi^{(1)}$ ), $\mu_{0}$ is the permeability of free space, and $\vec{P}_{N L}$ is the nonlinear part of 2), related to $\chi^{(3)}$. Closed form solutions of this equation are in general difficult to obtain, resulting in most techniques adopting simplifying assumptions.

Among the most popular approximations are the Slowly Varying Envelope Approximation (SVEA) [4], the Beam Propagation Method (BPM) [7], and the Nonlinear Shrödinger Equation (NLSE) [8]. However, these methods generally struggle with higher order nonlinearities or dispersion, may require slowly varying structures, may impose restrictions on how quickly material parameters can change, and may depend on guided modes or propagation restricted to a relatively small angular area [5]. As a result, often only a full solution of (3) can yield the complete behaviour of a given system. For instance, Joseph \& Taflove [9] found several instances in which the NLSE disagreed with a method which solved (3) directly.

Given these potential shortcomings, the best way to ensure the full response of an electrically complex material is captured is via a method which solves the nonlinear Maxwell's equations directly, such as those discussed in the next section.

\section{B. Numerical Methods for the Nonlinear Wave Equation}

One of the first techniques adapted to solve (3) directly in the time-domain was the Finite-Difference Time-Domain (FDTD) method [5]. The resulting algorithms proved simple, powerful, and, by solving the nonlinear wave equation directly in the time-domain, were capable of capturing the full nonlinear, dispersive, and wideband response of the system in a single computation. Unfortunately, these FDTD methods do also have some drawbacks, such as difficulties in modeling curved interfaces and boundaries, as well as sharp material discontinuities. Furthermore, the most popular FDTD algorithms tend to be explicit in time and as a result may incur reduced stability.

An alternative to FDTD which alleviates these concerns (at the cost of some added computational complexity) is the Finite-Element Time-Domain (FETD) method. In many respects, FETD is very similar to FDTD, and therefore it is unsurprising that existing generalizations of FETD to dispersive and nonlinear media utilize the same techniques as in FDTD. For instance, dispersion in FETD is generally treated using any of three standard techniques initially developed in FDTD: Recursive Convolution (RC) [10], Auxiliary Differential Equation (ADE) [11], and Z-transform [12], [13]. While each approach has been successful in its own right, the RC and ADE methods produce formulations that rapidly become intractable as the dispersive order of the material is increased. Z-transform-based methods, by comparison, tend to produce relatively simpler update schemes which remain so for higher dispersive orders. It is for these reasons that the z-transform technique will be employed in this paper, as further discussed in Section III-B.

Progress in nonlinear FETD has, however, not seen as widespread development or documentation. Instantaneous (nondispersive) nonlinear methods have been devised for nonlinear permeability and magnetic hysteresis [14], [15], which is useful in magnetic machines. Additionally, problems involving nonlinear conductivity and dielectric breakdown have also received attention [16], [17], though here again the permittivity is assumed to be linear. In contrast, a parametric quadratic programming method for piecewise linear permittivities has been presented in [18]. At present, this is one of very few papers to address instantaneous nonlinear dielectrics in FiniteElements, though it does not address the need to model arbitrary nonlinear functions.

As for modeling dispersive nonlinearities with FiniteElements, at present only the method presented in [19], an explicit mixed method using the ADE formalism for hexahedral elements, has been reported. While this does represent quite a general formulation, the use of the ADE technique for the permittivity rather than the more straightforward z-transform leads to increased complexity for high order dispersion. Moreover, since explicit time stepping is used, the scheme may prove less stable. Indeed, the authors reported that in many cases, time steps of at least half the linear Courant-FriedrichsLewy (CFL) condition were needed for stability. Furthermore, the method does not specifically treat linear dispersion or instantaneous nonlinearity. Lastly, the method's efficiency relies primarily on the use of hexahedral elements rather than the more popular triangular and tetrahedral elements.

In contrast, the method proposed in this paper aims to deal with both dispersion and dielectric nonlinearity in a versatile and easily generalizable way. For the first time, both instantaneous and dispersive nonlinear algorithms will be derived for the implicit mixed FETD method, offering increased geometric freedom, triangular elements, increased scalability, and stability. Moreover, by leveraging existing work on linear z-transform methods, the presented algorithm easily generalizes to arbitrary dispersive and nonlinear orders. 
The resulting scheme is among the first to comprehensi-bly model linear dispersion, instantaneous nonlinearity, and dispersive nonlinearities, all within a single Finite-Element simulation.

\section{DERIVATION}

\section{A. The Mixed FETD Method}

There are two main variants of the FETD method in use: second order vector wave equation (VWE) formulations [20] and mixed formulations [21]. As the name implies, the former approach solves the vector wave equation for the electric field only, using edge elements to represent the field. In this paper, the focus will be on the latter mixed formulation, which solves the first order coupled Maxwell's equations for both the electric and magnetic fields, using both edge and face elements. Even though the VWE technique is the more popular, the mixed approach does have advantages. For instance, it does not suffer from late time growth as the VWE can. Additionally, it has been posited that, under certain conditions, the two methods are homologous [13]. The mixed formulation presented here can therefore be easily adapted to the VWE in the future.

To begin, Faraday and Ampère's laws are expressed for linear non-dispersive media:

$$
\begin{aligned}
\nabla \times \vec{E} & =-\frac{\partial \vec{B}}{\partial t} \\
\nabla \times \frac{\vec{B}}{\mu_{0}} & =\vec{J}+\frac{\partial \vec{D}}{\partial t}
\end{aligned}
$$

where $\vec{B}$ is the magnetic flux density, $\vec{J}$ is a source volume current density, and in the linear non-dispersive case, $\vec{D}=\epsilon \vec{E}$. After meshing the domain, Whitney triangular or tetrahedral 1forms (edge elements) and 2-forms (face elements) can be used as basis functions to express the electric and magnetic fields within each element [22], [23]. These can then be used within a standard weak variational form of (4) and (5), producing the following spatially discretized linear systems:

$$
\begin{aligned}
{[C]^{T}\{e\} } & =-\frac{\partial\{b\}}{\partial t} \\
{[C]\left[M_{f}\right]\{b\} } & =[M] \frac{\partial\{e\}}{\partial t}+\{g\}
\end{aligned}
$$

in which brackets denote matrices, braces denote vectors, and the elemental matrices and source term are given by:

$$
\begin{aligned}
{\left[M_{f}\right]_{i j} } & =\int_{\Omega} \frac{1}{\mu_{0}} \vec{W}_{i}^{(2)} \cdot \vec{W}_{j}^{(2)} d \Omega \\
{[M]_{i j} } & =\int_{\Omega} \epsilon \vec{W}_{i}^{(1)} \cdot \vec{W}_{j}^{(1)} d \Omega \\
\{g\}_{i} & =\int_{\Omega} \vec{J} \cdot \vec{W}_{i}^{(1)} d \Omega
\end{aligned}
$$

where $\vec{W}^{(n)}$ represents a vector $\mathrm{n}$-form. It is also well known that the curl of the 1-forms is a subset of the 2-forms, and as a result, $\nabla \times \vec{W}_{i}^{(1)}$ can be written as a linear combination of $\vec{W}_{j}^{(2)}[24]$. Correspondingly, the $[C]$ matrix is composed solely of $\pm 1 \mathrm{~s}$ and $0 \mathrm{~s}$ and relates the two according to their connectivity: $\nabla \times \vec{W}_{i}^{(1)}=\sum_{j}[C]_{i, j} \vec{W}_{j}^{(2)}$.
From here, any number of temporal discretizations may be used. One popular variant uses central differences, resulting in a leap-frog scheme similar to the FDTD method [13]. While all the work presented in this paper is also applicable to this method, it is unfortunately only conditionally stable. However, one other possibility is the use of the Crank-Nicolson method, which when used with mixed FETD has recently been posited to be unconditionally stable [13]. Applying Crank-Nicolson to (6) and (7) yields:

$$
\begin{aligned}
\frac{\{b\}^{n+1}-\{b\}^{n}}{\Delta t} & =-\frac{1}{2}[C]\left(\{e\}^{n+1}+\{e\}^{n}\right) \\
{[M] \frac{\{e\}^{n+1}-\{e\}^{n}}{\Delta t}=} & \frac{1}{2}[C]^{T}\left[M_{f}\right]\left(\{b\}^{n+1}+\{b\}^{n}\right) \\
- & \frac{1}{2}\left(\{g\}^{n+1}+\{g\}^{n}\right) .
\end{aligned}
$$

From here, (11) can be used to eliminate $\{b\}^{n+1}$ from (12), which after simplification gives the following update equations for both the electric and magnetic fields:

$$
\begin{gathered}
\left([M]+\frac{\Delta t^{2}}{4}[C]^{T}\left[M_{f}\right][C]\right)\{e\}^{n+1}= \\
\left([M]-\frac{\Delta t^{2}}{4}[C]^{T}\left[M_{f}\right][C]\right)\{e\}^{n} \\
+\Delta t[C]^{T}\left[M_{f}\right]\{b\}^{n}-\frac{\Delta t}{2}\left(\{g\}^{n+1}+\{g\}^{n}\right) \\
\{b\}^{n+1}=-\frac{\Delta t}{2}[C]\left(\{e\}^{n+1}+\{e\}^{n}\right)+\{b\}^{n} .
\end{gathered}
$$

The solution process now involves two major steps:

1) Using (13) and known quantities from the previous time step, solve for $\{e\}^{n+1}$.

2) With $\{e\}^{n+1}$ known, use 14 to solve for $\{b\}^{n+1}$.

3) Repeat until the desired end time.

\section{B. Linear Dispersion}

As detailed in Section II-B, a well developed literature exists surrounding the treatment of linear dispersion within computational electromagnetics. While each of the previously discussed techniques has achieved notable success, the $\mathrm{z}$ transform approach exhibits several additional attractive properties, such as simplicity and the ability to easily generalize to high dispersive orders. For this reason, the following nonlinear algorithms will leverage the z-transform technique in their derivation. Specifically, the method initially derived in [13] for linear media will be briefly detailed here as the foundation for the nonlinear dispersive algorithms to follow.

As outlined earlier, having a dispersive medium implies that the permittivity is now a function of time, requiring the computation of a convolution:

$$
\begin{aligned}
\vec{D} & =\epsilon_{0} \vec{E}+\epsilon_{0} \chi^{(1)} * \vec{E} \\
& =\epsilon_{L} * \vec{E} .
\end{aligned}
$$

It can be shown that after spatial discretizations have been applied, the convolution which must be advanced in time is of the form:

$$
\{\mathcal{L}\}(t) \triangleq \epsilon_{L}(t) *[\tilde{M}]\{e\}(t)
$$


where $[\tilde{M}]$ is defined similarly to $[M]$ in $[9$, except for the lack of the permittivity:

$$
[\tilde{M}]_{i j}=\int_{\Omega} \vec{W}_{i}^{(1)} \cdot \vec{W}_{j}^{(1)} d \Omega .
$$

To alleviate this burden, a z-transform approach may be adopted, in which the convolution is instead converted into a series of multiplications. The Laplace transform of 17p can be taken, immediately converting the convolution into a multiplication. Generally, the resulting expression for $\epsilon_{L}(s)$ will be the quotient of two polynomials:

$$
\{\mathcal{L}\}(s)=\epsilon_{L}(s)[\tilde{M}]\{e\}(s)=\frac{c_{p} s^{p}+\ldots+c_{0}}{d_{p} s^{p}+\ldots+d_{0}}[\tilde{M}]\{e\}(s) .
$$

The key step is to then apply a bilinear Möbius transform, to map from the $s$-domain to the discrete $z$-domain:

$$
s \mapsto \frac{2}{\Delta t} \frac{1-z^{-1}}{1+z^{-1}} .
$$

Applying this transformation to [19], normalizing the first term in the denominator, and cross multiplying gives:

$$
\left(1+\ldots+b_{p} z^{-p}\right)\{\mathcal{L}\}(z)=\left(a_{0}+\ldots+a_{p} z^{-p}\right)[\tilde{M}]\{e\}(z) .
$$

By leveraging the time shifting property of the z-transform, (21) can be transformed back to the time domain, yielding an update equation for the convolution:

$$
\begin{aligned}
\{\mathcal{L}\}^{n}=a_{0}[\tilde{M}]\{e\}^{n} & +\ldots+a_{p}[\tilde{M}]\{e\}^{n-p} \\
& -b_{1}\{\mathcal{L}\}^{n-1}-\ldots-b_{p}\{\mathcal{L}\}^{n-p} .
\end{aligned}
$$

Rather than explicitly storing past field and convolution values, the Transposed Direct Form II method suggested in [13] can be used. In essence, rather than explicitly storing past values, they are accumulated into auxiliary variables as time stepping progresses, with the number of required auxiliary variables depending on the amount of history needed (itself controlled by the dispersive order $p$ ):

$$
\begin{array}{rlrl}
\{\mathcal{W}\}_{\alpha}^{n} & =a_{\alpha}[\tilde{M}]\{e\}^{n}-b_{\alpha}\{\mathcal{L}\}^{n}+\{\mathcal{W}\}_{\alpha+1}^{n-1} & & \alpha<p \\
\{\mathcal{W}\}_{\alpha}^{n} & =a_{\alpha}[\tilde{M}]\{e\}^{n}-b_{\alpha}\{\mathcal{L}\}^{n} & & \alpha=p \\
\{\mathcal{L}\}^{n} & =a_{0}[\tilde{M}]\{e\}^{n}+\{\mathcal{W}\}_{1}^{n-1} . &
\end{array}
$$

The modified form of (13) can now be obtained, incorporating the dispersion, by substituting the convolution at a given time step with (25):

$$
\begin{aligned}
& \left(a_{0}[\tilde{M}]+\frac{\Delta t^{2}}{4}[C]^{T}\left[M_{f}\right][C]\right)\{e\}^{n+1}= \\
& \left(a_{0}[\tilde{M}]-\frac{\Delta t^{2}}{4}[C]^{T}\left[M_{f}\right][C]\right)\{e\}^{n}+\Delta t[C]^{T}\left[M_{f}\right]\{b\}^{n} \\
& -\left(\{\mathcal{W}\}_{1}^{n}-\{\mathcal{W}\}_{1}^{n-1}\right)+\frac{1}{2} \Delta t\left(\{g\}^{n+1}+\{g\}^{n}\right) .
\end{aligned}
$$

The solution procedure is now quite similar to before, with the exception of the need to also update the auxiliary variables:

1) Using (26) and all known values from the previous time step, obtain $\{e\}^{n+1}$.

2) Using this result, obtain $\{b\}^{n+1}$ using (14).

3) Advance each of the auxiliary variables and the convolution to $n+1$ in order, using (23) - 25).

4) Repeat until desired end time.

\section{Instantaneous Nonlinearity}

In the case of an instantaneous nonlinearity, convolutions are absent; however the permittivity now depends on the electric field strength $(\alpha=0)$ :

$$
\vec{D}=\underbrace{\epsilon_{0}\left(1+\chi^{(1)}+\chi^{(3)} E^{2}\right)}_{\epsilon} \vec{E} .
$$

As mentioned earlier, this type of problem has not received much treatment in the literature, and so the following derivation serves as an important foundation of FETD, both for instantaneous nonlinearity, and the dispersive nonlinearities to follow. The spatial discretization of (5) remains much the same, except that the matrix $[M]$ is no longer constant in time due to its dependence on $E$ :

$$
\begin{aligned}
{[M]_{i j} } & =\int_{\Omega} \epsilon(E) \vec{W}_{i}^{(1)} \cdot \vec{W}_{j}^{(1)} d \Omega \\
& =\int_{\Omega} \epsilon_{0}\left(1+\chi^{(1)}+\chi^{(3)} E^{2}\right) \vec{W}_{i}^{(1)} \cdot \vec{W}_{j}^{(1)} d \Omega
\end{aligned}
$$

and must remain within the temporal derivative:

$$
\frac{\partial}{\partial t}[M]\{e\} \approx \frac{[M]^{n+1}\{e\}^{n+1}-[M]^{n}\{e\}^{n}}{\Delta t} .
$$

The resulting update equation for the electric field appears identical to that in (13), but it is now nonlinear thanks to 30):

$$
\begin{aligned}
&\left([M]^{\mathbf{n}+\mathbf{1}}\right.\left.+\frac{\Delta t^{2}}{4}[C]^{T}\left[M_{f}\right][C]\right)\{e\}^{n+1}= \\
&\left([M]^{\mathbf{n}}-\frac{\Delta t^{2}}{4}[C]^{T}\left[M_{f}\right][C]\right)\{e\}^{n} \\
&+\Delta t[C]^{T}\left[M_{f}\right]\{b\}^{n}-\frac{1}{2}\left(\{g\}^{n+1}+\{g\}^{n}\right)
\end{aligned}
$$

and should be solved via an iterative method such as NewtonRaphson.

In the Newton-Raphson method, a system of nonlinear equations of the form $\{f\}=0$, dependent on a set of variables $\{x\}$, may be solved by iterating in the following manner [14]:

$$
\{x\}_{(k+1)}=\{x\}_{(k)}-[J]^{-1}\{f\}_{(k)}
$$

in which $(k)$ is the iteration number, and $[J]$ is the Jacobian matrix defined as:

$$
[J]_{i j}=\frac{\partial\{f\}_{i}}{\partial\{x\}_{j}} .
$$

By moving all terms of (31) to one side, the NewtonRaphson method can easily be applied, assuming the Jacobian can be found. The derivation of the Jacobian that follows is very similar to that presented in [14] for nonlinear magnetic materials and produces similar expressions.

Here, the unknown variable of interest is the vector $\{e\}^{n+1}$, and so the Jacobian will only take derivatives with respect to this quantity. As a result, only the left-hand side of (31) will 
have a non-zero contribution to the Jacobian. Expressing the matrix multiplication with $\{e\}^{n+1}$ explicitly yields:

$$
\begin{aligned}
& {[J]_{i j}=\frac{\partial\{f\}_{i}}{\partial\{e\}_{j}^{n+1}}=} \\
& \frac{\partial}{\partial\{e\}_{j}^{n+1}} \sum_{k}\left([M]_{i k}^{n+1}+\frac{\Delta t^{2}}{4}[C]^{T}\left[M_{f}\right][C]_{i k}\right)\{e\}_{k}^{n+1}
\end{aligned}
$$

Distributing the derivative into the sum and making use of the product rule produces:

$[J]_{i j}=[M]_{i j}^{n+1}+\frac{\Delta t^{2}}{4}[C]^{T}\left[M_{f}\right][C]_{i j}+\sum_{k} \frac{\partial[M]_{i k}^{n+1}}{\partial\{e\}_{j}^{n+1}}\{e\}_{k}^{n+1}$.

This last term can be further refined by returning to the definition of $[M]$ and bringing the derivatives and sum inside the integral, in addition to using the chain rule:

$$
\int_{\Omega} \sum_{k} \frac{\partial \epsilon^{n+1}}{\partial E^{n+1}} \frac{\partial E^{n+1}}{\partial\{e\}_{j}^{n+1}} \vec{W}_{i}^{(1)} \cdot \vec{W}_{k}^{(1)}\{e\}_{k}^{n+1} d \Omega .
$$

Recalling that within each element, the electric field is represented as:

$$
\vec{E}^{n+1}=\sum_{k} \vec{W}_{k}^{(1)}\{e\}_{k}^{n+1}
$$

permits a further simplification to:

$$
\int_{\Omega} \frac{\partial \epsilon^{n+1}}{\partial E^{n+1}} \frac{\partial E^{n+1}}{\partial\{e\}_{j}^{n+1}} \vec{W}_{i}^{(1)} \cdot \vec{E}^{n+1} d \Omega
$$

Lastly, using (37), it can be shown via straightforward computation that:

$$
\frac{\partial E^{n+1}}{\partial\{e\}_{j}^{n+1}}=\vec{W}_{j}^{(1)} \cdot \frac{\vec{E}^{n+1}}{E^{n+1}} .
$$

Making this final substitution produces the desired compact form of the Jacobian:

$$
\begin{aligned}
& {[J]_{i j}=[M]_{i j}+\frac{\Delta t^{2}}{4}[C]^{T}\left[M_{f}\right][C]_{i j}} \\
& +\int_{\Omega} \frac{1}{E^{n+1}} \frac{\partial \epsilon^{n+1}}{\partial E^{n+1}}\left(\vec{W}_{i}^{(1)} \cdot \vec{E}^{n+1}\right)\left(\vec{W}_{j}^{(1)} \cdot \vec{E}^{n+1}\right) d \Omega
\end{aligned}
$$

where in the present case:

$$
\frac{\partial \epsilon^{n+1}}{\partial E^{n+1}}=2 \epsilon_{0} \chi^{(3)} E^{n+1} .
$$

With this result, the solution may now be found in a straightforward, albeit computationally intensive, manner. While the Jacobian in 40 is symmetric, it will in general change during each iteration of (32), as will the $[M]$ matrix in $[29)$. They will therefore need to be recomputed within each nonlinear element and globally reassembled multiple times within each time step.

The general solution procedure is now as follows:

1) Iterate (31) using (32) and (40), recomputing and assembling $[J]$ and $[M]$ each time, until $\{e\}^{n+1}$ converges to the desired tolerance.

2) Compute $\{b\}^{n+1}$ using 14 .

3) Repeat the process until the desired end time.

\section{Dispersive Nonlinearity}

Attention is now turned toward the most general form of the permittivity, including linear dispersion, instantaneous nonlinearity, as well as dispersive nonlinearity:

$$
\vec{D}=\epsilon_{L} * \vec{E}+\epsilon_{0} \chi^{(3)}\left(\alpha E^{2}+(1-\alpha) g(t) * E^{2}\right) \vec{E} .
$$

Such a general formulation is nascent within the FETD method, and the ensuing novel approach is quite versatile. When (42) is inserted into (5), and the spatial discretization applied, the resulting equation contains terms of the following form:

$$
\epsilon_{L} *[\tilde{M}]\{e\}+[\hat{M}]\{e\}
$$

where $[\tilde{M}]$ is the same as in $[18$ and $[\hat{M}]$ is given by:

$[\hat{M}]_{i j}=\int_{\Omega} \epsilon_{0} \chi^{(3)}\left(\alpha E^{2}+(1-\alpha) g(t) * E^{2}\right) \vec{W}_{i}^{(1)} \cdot \vec{W}_{j}^{(1)} d \Omega$.

The first term in (43) is clearly a result of the linear dispersion and is identical to that obtained previously in (17), while the second term encapsulates the nonlinearity and is of the same form as 29). The linear dispersive term can be treated identically as before and produces the same auxiliary variable update procedure, transforming (43) into:

$$
a_{0}[\tilde{M}]\{e\}^{n+1}+\{\mathcal{W}\}_{1}^{n}+[\hat{M}]^{n+1}\{e\}^{n+1}
$$

or, defining $[K]=a_{0}[\tilde{M}]+[\hat{M}]$ :

$$
[K]^{n+1}\{e\}^{n+1}+\{\mathcal{W}\}_{1}^{n} \text {. }
$$

This $[K]$ matrix, however, now contains a convolution for which an update procedure must also be derived. Using the same procedure as before, the $\mathrm{z}$-transform can be used to generate an update equation for $\mathcal{B} \triangleq g(t) * E^{2}$ :

$$
\begin{aligned}
\mathcal{B}^{n}=h_{0}\left(E^{2}\right)^{n}+\ldots+ & h_{q}\left(E^{2}\right)^{n-q} \\
& -w_{1} \mathcal{B}^{n-1}-\ldots-w_{q} \mathcal{B}^{n-q} .
\end{aligned}
$$

Furthermore, a set of auxiliary variables can also be introduced to facilitate the update as before:

$$
\begin{array}{lll}
\mathcal{G}_{\alpha}^{n}=h_{\alpha}\left(E^{2}\right)^{n}-w_{\alpha} \mathcal{B}^{n}+\mathcal{G}_{\alpha+1}^{n-1} & & \alpha<q \\
\mathcal{G}_{\alpha}^{n}=h_{\alpha}\left(E^{2}\right)^{n}-w_{\alpha} \mathcal{B}^{n} & & \alpha=q \\
\mathcal{B}^{n} & =h_{0}\left(E^{2}\right)^{n}+\mathcal{G}_{1}^{n-1} . &
\end{array}
$$

Given this convolution is a scalar function, as opposed to the vector quantity used in the linear case, it can be directly substituted into the $[K]$ matrix at the required time step using (50), yielding:

$$
\begin{array}{r}
{[K]_{i j}^{n+1}=\int_{\Omega}\left(a_{0}+\alpha \epsilon_{0} \chi^{(3)}\left(E^{2}\right)^{n+1}+(1-\alpha) \epsilon_{0} \chi^{(3)}\right.} \\
\left.\left[h_{0}\left(E^{2}\right)^{n+1}+\mathcal{G}_{1}^{n}\right]\right) \vec{W}_{i}^{(1)} \cdot \vec{W}_{j}^{(1)} d \Omega
\end{array}
$$

with the final update equation for $\vec{E}$ given as:

$$
\begin{aligned}
& \left([K]^{n+1}+\frac{\Delta t^{2}}{4}[C]^{T}\left[M_{f}\right][C]\right)\{e\}^{n+1}= \\
& \left([K]^{n}-\frac{\Delta t^{2}}{4}[C]^{T}\left[M_{f}\right][C]\right)\{e\}^{n}+\Delta t[C]^{T}\left[M_{f}\right]\{b\}^{n} \\
& -\left(\{\mathcal{W}\}_{1}^{n}-\{\mathcal{W}\}_{1}^{n-1}\right)+\frac{1}{2} \Delta t\left(\{g\}^{n+1}+\{g\}^{n}\right) .
\end{aligned}
$$


The derivation of the Jacobian proceeds in much the same way as it did before. Indeed, the derivation presented in the previous section can be repeated replacing the $[M]$ matrix with the $[K]$ matrix, leading to the same Jacobian expression as in (40), except for a change in (41):

$$
\frac{\partial \epsilon^{n+1}}{\partial E^{n+1}}=2 \epsilon_{0} \chi^{(3)} E\left(\alpha+(1-\alpha) h_{0}\right) .
$$

The general solution procedure is now as follows:

1) Iterate (52), using (32) and (40), re-computing and assembling the $[J]$ and $[K]$ matrices each time, until $\{e\}^{n+1}$ converges to the desired tolerance.

2) Compute $\{b\}^{n+1}$ using (14).

3) Update the linear auxiliary variables using (23) (25) to $n+1$.

4) Update the nonlinear auxiliary variables using (48) to $n+1$.

5) Repeat the process until the desired end time.

\section{IMPLEMENTATION}

In this section, a few of the specific details concerning implementation of the above algorithms are discussed and presented.

\section{A. Newton-Raphson Iteration}

Given the computational cost associated with nonlinear iteration, it is important to attempt to minimize the number of iterations required to achieve convergence. One way to do this is to choose the initial solution guess $\{e\}_{(0)}^{n+1}$ to be as close as possible to the true solution.

If the time step $\Delta t$ chosen in the FETD update procedure above is sufficiently small, then the solution at time $n+1$ should be relatively close to that at $n$. As a result, a natural choice for a starting guess is the previous time step's solution [25]:

$$
\{e\}_{(0)}^{n+1}=\{e\}^{n} .
$$

\section{B. Evaluation of Elemental Matrices}

For many linear elements, the entries of the $[M]$ matrix can be found analytically and tabulated for easy use [20]. In the nonlinear case, however, the dependence of $\epsilon$ on $\{e\}$ no longer permits such simple formulae. In most cases, this leaves little choice but to numerically compute the entries of each nonlinear elemental $[M],[J]$, and $[K]$ matrix. Luckily, Gaussian quadrature rules can be found for triangles and tetrahedra to quite high order [20], facilitating the integrations. In particular, quadrature rules given in terms of simplex coordinates easily permit the computation of the requisite 1forms $\vec{W}_{i}^{(1)}$ in 51 .

Moreover, since the nonlinearity introduces a dependance on the unknown variables, additional code and data structures are required beyond the usual geometric mesh data. For instance, each element is required to monitor its associated edge values and basis functions, and generate the field strength at each quadrature point within the simplex.

\section{Auxiliary Variable Updating}

While the update procedure for the linear convolution auxiliary variables in (23) (25) is relatively straightforward, those for the nonlinear portion in (48)-(50) are less so. For instance, while the nonlinear convolution may produce a scalar result, as opposed to the vector result in the linear case, it is located within the integral for $[K]$ in (51). As such, in order to be numerically integrated, as discussed in the last section, the convolution must be known at each of the quadrature points, within each element.

The simplest approach is to store the convolution values at each quadrature point, within each nonlinear element, for as many past time steps are required for the current dispersive order $q$. The auxiliary update equations are then applied to every quadrature point, for each time step. The resulting implementation trades space for simplicity.

However, an alternative may be possible in which the convolution is itself expanded in terms of scalar basis functions. This would have the benefit of only needing to store and advance the expansion weights, at the cost of now interpolating the convolution at each quadrature point. Care must be taken, however, such that the accuracy of the convolution representation not affect the global solution accuracy.

Such an expansion of the convolution (or even of the permittivity itself) may introduce an additional advantage. If the same scalar simplex interpolation functions are used for $\epsilon$ as are used in the construction of the edge and face elements [23], closed-form analytic expressions for the nonlinear matrices can be recovered. For example, one well-known formula evaluates the integral of products of simplicial basis functions as [20]:

$$
\int_{\Omega} N_{1}^{l} N_{2}^{m} N_{3}^{n} d \Omega=\frac{l ! m ! n !}{+m+n+2) !} 2 \Delta .
$$

A possible complication arising from this method, however, is in allowing the permittivity to be discontinuous across material boundaries. While solutions to this are possible, they may result in increased complexity. These interpolation methods were not employed in this paper and are suggested as possible alternatives or future improvements.

\section{Stability}

When formulating a numerical method, an important consideration is that of stability. In the case of linear materials, with or without dispersion, the above implicit mixed CrankNicolson scheme has been posited to be unconditionally stable via an equivalence with the Newmark- $\beta$ method [13].

Unfortunately, unlike in the linear case, there are few general techniques available to determine the stability of a nonlinear numerical method. Attempts at analyzing the above algorithms have not yet provided any insight into their stability. Moreover, while it is hoped that the unconditional stability of the underlying linear method is maintained in the nonlinear case, there is no guarantee that this will occur. Nevertheless, for all numerical tests performed in the generation of the data presented in the next section, no instabilities were found. 


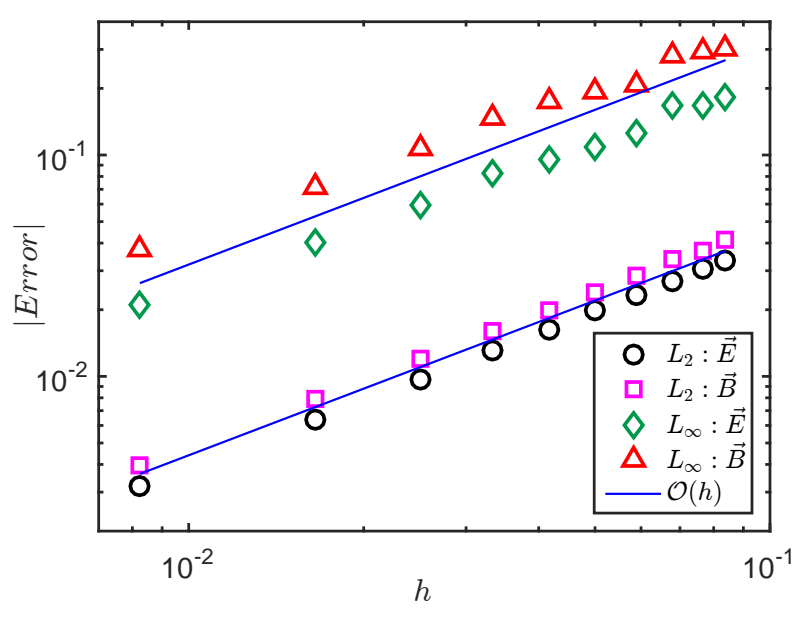

Fig. 1. Convergence in the $L_{2}$ and $L_{\infty}$ norms for the nonlinear dispersive Crank-Nicolson mixed FETD method.

\section{RESULTS}

In this section, the algorithms presented in the previous sections will be benchmarked to verify their accuracy and convergence. Furthermore, the methods will be used to demonstrate the occurrence of some well-known nonlinear phenomena.

\section{A. Convergence}

As mentioned earlier, the nonlinear wave equation (3) is in general very difficult to solve exactly in closed form. As a result, it can be challenging to judge the accuracy of a new numerical method without a known test case. For this reason, an approach is here adopted in which a solution is artificially manufactured. An arbitrary function is first selected to be the exact solution, to which the differential equation of interest is then applied. Since this function will not solve the equation exactly, there will naturally be a residual. By selecting a source term which exactly equals this residual, the selected function becomes the exact solution. A simulation can then be run with this source term, and the computed solution compared to the selected exact one, yielding convergence and accuracy data.

In the present case, the sample problem studied was that of a simple unit square domain in two spatial dimensions, $\Omega=[0,1] \times[0,1]$, in which perfect electric conductor (PEC) boundary conditions were imposed on each of the four edges. Triangular elements were used to mesh the area, and the manufactured exact solutions for the $\vec{E}$ and $\vec{B}$ fields were selected as follows:

$$
\begin{aligned}
& \vec{E}=\left\{\begin{array}{cc}
-\sin (2 \pi x) \sum_{n=1}^{3} a_{n} n \sin (n \omega t) \hat{a}_{y} & 0 \leq t \leq T \\
0 & \text { otherwise }
\end{array}\right. \\
& \vec{B}=\left\{\begin{array}{cc}
-\frac{2 \pi}{\omega} \cos (2 \pi x) \sum_{n=0}^{3} a_{n} \cos (n \omega t) \hat{a}_{z} & 0 \leq t \leq T \\
0 & \text { otherwise }
\end{array}\right.
\end{aligned}
$$

where:

$$
a_{0}=\frac{3179}{9000} a_{1}=-\frac{4392}{9000} a_{2}=\frac{1305}{9000} a_{3}=-\frac{92}{9000}
$$

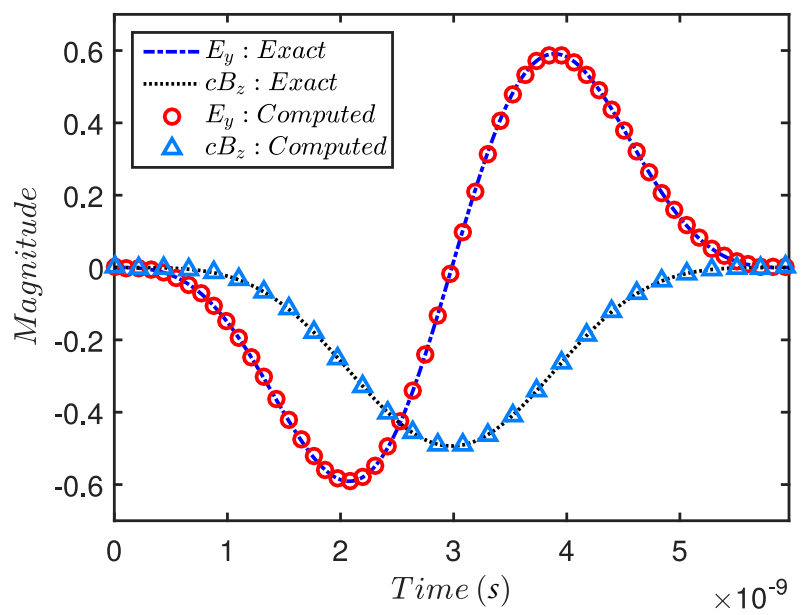

Fig. 2. Computed and exact solutions as functions of time.

and $\omega \approx 1.0530 \times 10^{9} \mathrm{rad} / \mathrm{s}$, such that the temporal shape of the $\vec{E}$ and $\vec{B}$ fields are the Differentiated Blackman-Harris and Blackman-Harris pulses, respectively.

Since the linear dispersive algorithm has already been verified in [13], the test permittivity presented here contains only the nonlinear dispersive term $(\alpha=0)$ :

$$
\epsilon=\epsilon_{0}\left(1+\chi^{(1)}+\chi^{(3)} g(t) * E^{2}\right)
$$

where $\chi^{(1)}=2.2, \chi^{(3)}=4.1$, and $g(t)$ was chosen to represent a first order Debye-like dispersion:

$$
g(t)=e^{t / \tau_{e}}
$$

with $\tau_{e}=10^{-9} \mathrm{~s}$.

While an instantaneous nonlinearity is not included in the present results, its accuracy was also verified with a similar outcome. Lastly, the selected permittivity parameters above (and in all subsequent simulations) were chosen and scaled either to facilitate the calculation of the necessary source term or to have the phenomena of interest be readily observable within the given computational domain, and do not necessarily reflect any particular existing material. To that end, the required $\vec{J}$ term in (10) was determined via the procedure outlined above. While a closed form expression for $\vec{J}$ is obtainable via a rather tedious computation, the expression is quite large and so has been omitted for clarity.

The accuracy and convergence of the method were obtained by performing several computations on progressively refined spatial grids, with the temporal time step held fixed at $\Delta t=$ $h / c$, where $h$ is the average element side length in the mesh. The tolerance of the nonlinear Newton-Raphson stop condition was set to $10^{-6}$, and the error was measured in terms of the $L_{2}$ and $L_{\infty}$ norms, defined here as:

$$
\begin{aligned}
L_{2} & =\frac{1}{\sqrt{N_{t}}}\left[\sum_{n=1}^{N_{t}} \int_{\Omega}\left|\vec{E}^{n}-\vec{E}_{e x}^{n}\right|^{2} d \Omega\right]^{1 / 2} \\
L_{\infty} & =\max _{t, x}\left|\vec{E}-\vec{E}_{e x}\right|
\end{aligned}
$$



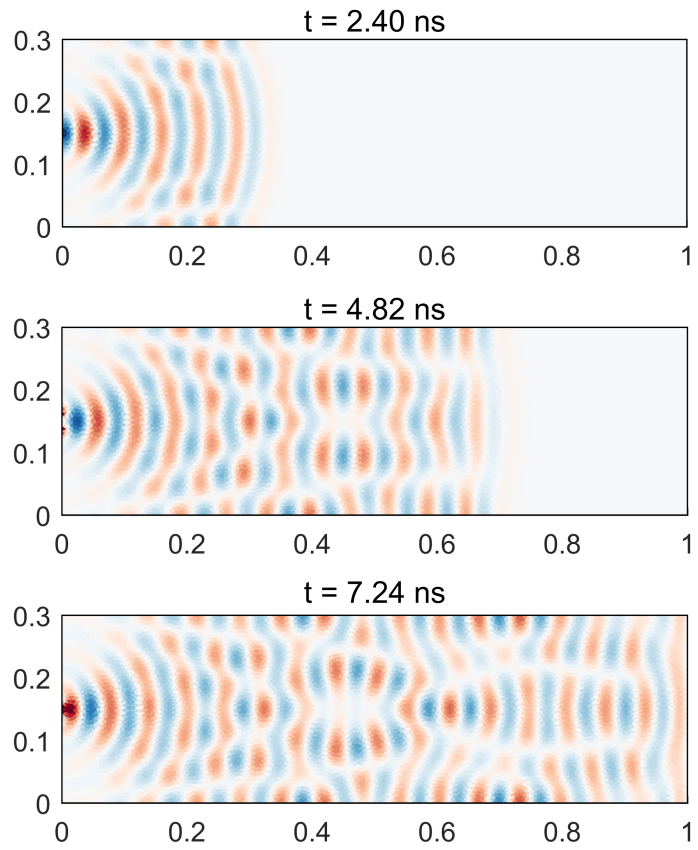

Fig. 3. Beam diffraction while propagating in a bulk linear medium.

where $\vec{E}$ is the computed solution and $\vec{E}_{e x}$ is the exact solution.

The convergence results are presented in Fig. 1 in which the error of both the $\vec{E}$ and $\vec{B}$ fields are plotted in the aforementioned norms. From this plot it is apparent that all four error traces are converging to first order in $h$ (and therefore also in $t$ ). While the Crank-Nicolson method is globally second order in time (like Newmark- $\beta$ ), this first order convergence is expected since the Whitney forms used in the representation of $\vec{E}$ and $\vec{B}$ here are only complete to zeroth order [20], thus lowering the global accuracy.

Additionally, in Fig. 2 both the exact and computed solutions are plotted as functions of time, for $h=0.0082$, at the point $(x, y)=(0.8325,0.7314)$. In both cases there is very good agreement between the computed and exact solutions, corroborating the trends of Fig. 1]

\section{B. Spatial and Temporal Solitons}

Having demonstrated the accuracy and convergence of the method in the previous section, here the algorithm is applied to a few physically significant problems, demonstrating the occurrence of some well-known nonlinear phenomena.

1) Spatial Soliton: In linear bulk media, as an electromagnetic wave propagates, it naturally diffracts and spreads out. As a result, any initial beam rapidly widens and loses intensity. In a nonlinear material, however, the dependence of the permittivity on the field strength can result in a lensing effect, negating the tendency for the beam to diffract. Given the correct combination of field strength, beam shape, and nonlinear susceptibility, the resulting self-focusing can permit the propagation of a confined beam over large distances within a homogeneous material, known as a spatial soliton.

To demonstrate the occurence of these two phenemona, a slab of bulk 2D rectangular media was simulated, in which an
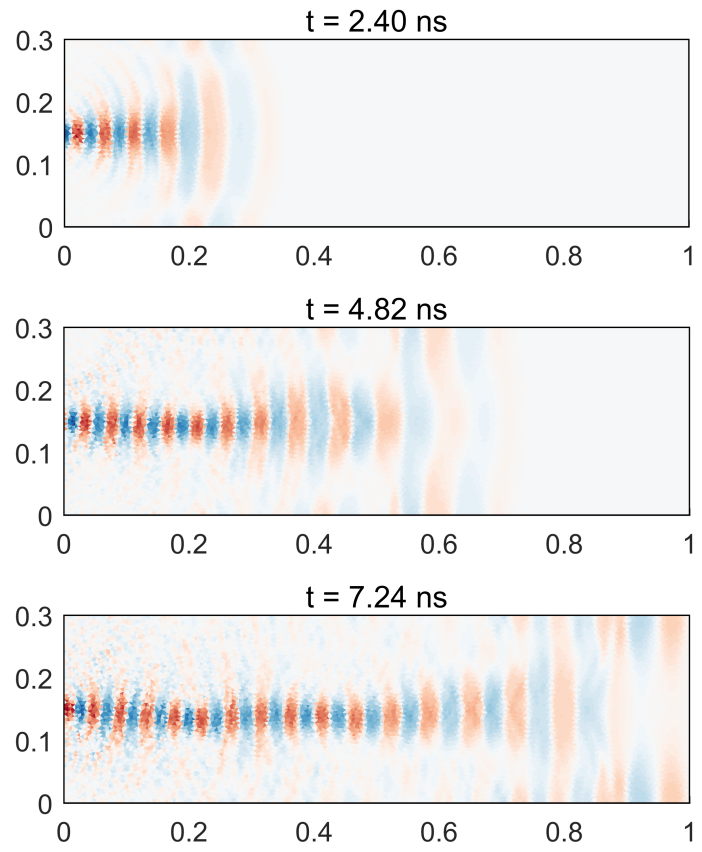

Fig. 4. Creation of a spatial soliton in a bulk nonlinear medium.

initial confined beam was injected on the left-hand side and allowed to propagate. The simulation domain was a rectangle $30 \mathrm{~cm}$ wide by $100 \mathrm{~cm}$ long, with PEC boundaries. The beam's transverse shape was that of a hyperbolic secant, with a full width at half maximum (FWHM) value of $1.46 \mathrm{~cm}$ and a frequency of $2.4 \mathrm{GHz}$. The case of a linear non-dispersive medium is demonstrated in Fig. 3 for $\epsilon_{L}=4.2$. Here the initial bright localized beam can be seen on the leftmost boundary, but rapidly spreads out over time, rebounding off the PEC upper and lower boundaries and creating a diffuse interference pattern.

In contrast, Fig. 4 shows the result of filling the domain with a material exhibiting an instantaneous nonlinearity $(\alpha=1)$, with $\chi^{(1)}=3.2$ and $\chi^{(3)}=1.5 \times 10^{-19} \mathrm{~m}^{2} / V^{2}$. The effect of nonlinearity is immediately apparent, as the beam has remained roughly confined to its original transverse shape during propagation. Note that in this simulation the beam intensity is ramped up from zero, and as a result the soliton is not established until the beam has reached an appropriate strength, leading to the initial diffuse wave front.

2) Temporal Soliton: Unlike the previous section, here attention is turned toward guided waves, such as those that exist within optical fibers and cables. As opposed to propagation in bulk media, the guiding structure ensures that the transverse shape of the wave is maintained during propagation. However, if linear dispersion is introduced, the wave or pulse can still become distorted. Since the permittivity is dependent on frequency, each spectral component of a propagating pulse travels at a different speed, resulting in a group delay which causes the pulse to broaden over time. On the other hand, if the material also exhibits nonlinearity, much like in the previous case, it is possible to tune the pulse parameters such that the linear dispersion is effectively cancelled out by the nonlinearity. The result is a pulse which does not change shape 


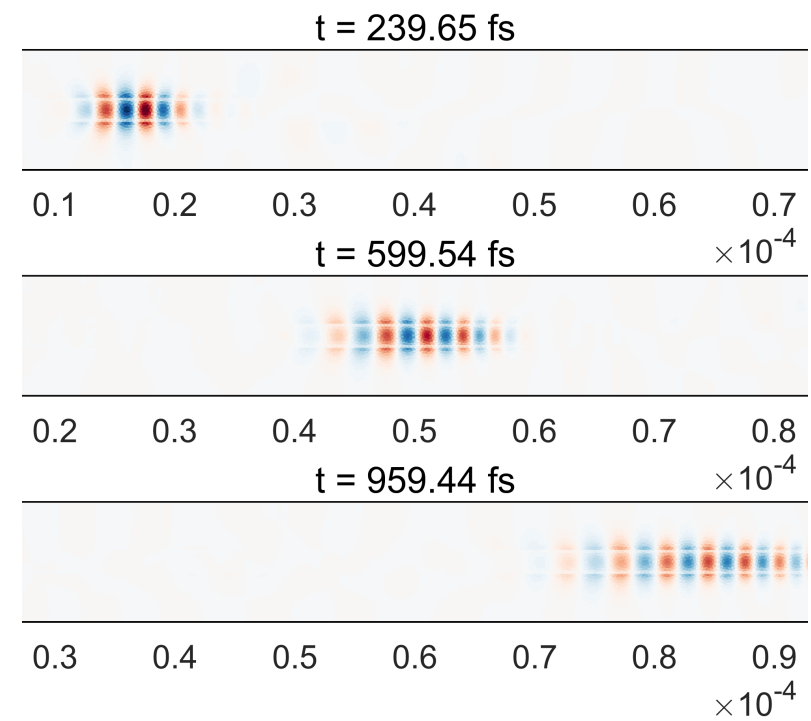

Fig. 5. The effects of anomalous linear dispersion, leading to pulse broadening over time.

as it propagates, known as a temporal soliton.

As before, a 2D problem was selected to recreate the behaviours described above. Rather than a bulk medium, a dielectric slab waveguide was chosen to more closely mimic the operation of an optical fiber. The rectangular domain measured $10 \mu \mathrm{m}$ wide by $100 \mu \mathrm{m}$ long, and was composed of three dielectric layers. The center dielectric measured $2 \mu \mathrm{m}$ wide, with the remaining areas being free space. The pulse was excited on the leftmost boundary in the fundamental TM mode, with a modulated hyperbolic secant envelope in time. The pulse envelope had a FWHM of approximately $52.7 \mathrm{fs}$ and a fundamental frequency of $50 \mathrm{THz}$. Approximately 6 periods of the carrier wave were contained within the pulse.

In both cases, a second order linear Lorentz dispersion was present, with the corresponding time-dependent linear susceptibility expressed as:

$\chi^{(1)}(t)=\chi_{\infty} \delta(t)+\frac{\left(\chi_{s}-\chi_{\infty}\right) \omega_{0}^{2}}{\sqrt{\omega_{0}^{2}-\delta^{2}}} e^{-\delta t} \sin \left(\sqrt{\omega_{0}^{2}-\delta^{2}} t\right) u(t)$

in which $\omega_{0}$ is the resonant frequency, $\delta$ is the damping constant, $u(t)$ is the unit step function, and $\delta(t)$ is the delta function. Additionally, the following values were selected to obtain easily observable pulse broadening over the distance simulated: $\omega_{0} \approx 1.885 \times 10^{14}, \delta=2 \times 10^{11}, \chi_{s}=6.1$, and $\chi_{\infty}=4.7$.

Fig. 5 shows the effects of the linear dispersion on the pulse. While initially compact, the pulse rapidly experiences anomalous linear dispersion, in which higher frequency components travel faster than their lower frequency counterparts, producing an increasingly distorted and stretched wave. This negative chirp is partially visible in Fig. 5 upon close inspection.

In the nonlinear case, not only was the above linear dispersion present, but also an instantaneous and dispersive nonlinearity. The nonlinear dispersive term $g(t)$ was also

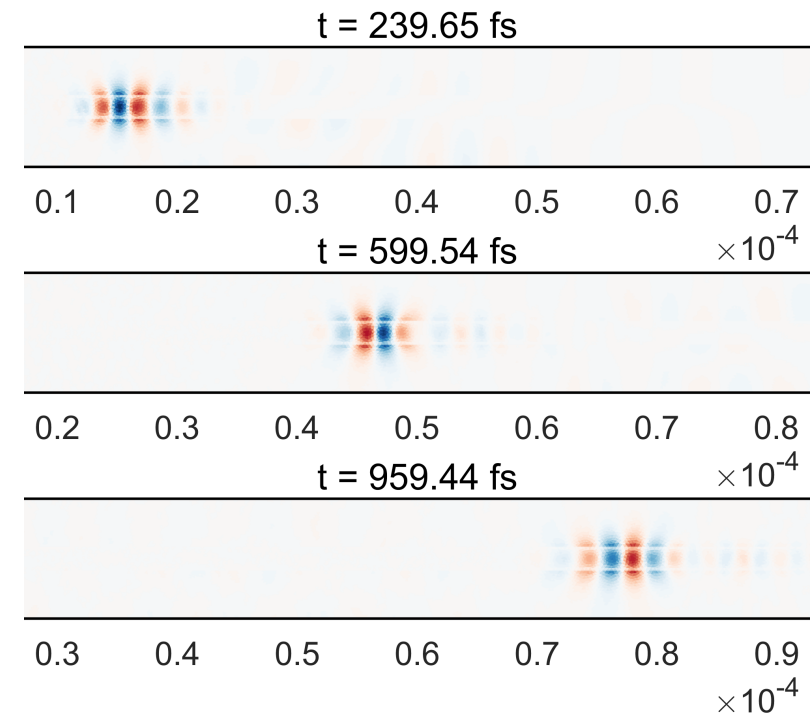

Fig. 6. Production of a temporal soliton within a dielectric slab waveguide.

selected to yield a Lorentzian-type dispersion:

$$
g(t)=\left(\frac{\tau_{1}^{2}+\tau_{2}^{2}}{\tau_{1} \tau_{2}^{2}}\right) e^{-t / \tau_{2}} \sin \left(\frac{t}{\tau_{1}}\right) u(t)
$$

where $\tau_{1}$ represents the optical phonon period, and $\tau_{2}$ the phonon lifetime [5]. In the present case, the following nonlinear values were selected: $\chi^{(3)}=1.1 \times 10^{-18} \mathrm{~m}^{2} / V^{2}, \alpha=0.7$, $\tau_{1}=3.358 \times 10^{-14} \mathrm{~s}$, and $\tau_{2}=1 \times 10^{-13} \mathrm{~s}$. Note that these values should result in the anomolous linear dispersion being stronger than its normal nonlinear counterpart.

Fig. 6 shows a dramatic contrast to Fig. 5, as a result of including nonlinear effects, resulting in the production of a temporal soliton. As the pulse propagates its shape and size remain roughly constant, with no significant chirping detected.

\section{CONCLUSION}

In conclusion, a novel mixed finite-element time-domain method for the simulation of nonlinear dispersive materials has been derived, verified, and used to demonstrate the occurrence of several well-known nonlinear phenomena. In contrast to existing methods, the presented technique is capable of handling the most general combinations of problem geometry and material behaviour, including linear dispersion, instantaneous nonlinearity, and dispersive nonlinearity. Moreover, by making use of the Crank-Nicolson mixed FETD formulation, the resulting scheme is in no danger of late-time growth, and has so far proven to be stable and accurate. The similarities between the Crank-Nicolson mixed formulation and the Newmark- $\beta$ VWE can also be leveraged in the future to easily produce a method which uses only edge elements.

By leveraging the Möbius transform method, the presented algorithm is quite versatile. While the simulations presented in this paper contained, respectively, only up to second and third order dispersion and nonlinearities, the generalization to higher dispersive and nonlinear orders is very straightforward. In the dispersive case, this results only in the introduction of additional auxiliary variables, while in the nonlinear case, 
only $\epsilon$ and $\partial \epsilon / \partial E$ are altered. It is also worth noting that while in this paper the permeability was assumed constant, the incorporation of magnetic dispersion would not pose any issue and would simply result in another set of auxiliary variables, as detailed in [13].

Lastly, one major issue in the application of the above method is the intense computational burden posed by the nonlinear iteration. The requirement for multiple matrix solves per time step, as well as the need to recompute and reassemble the elemental matrices, results in significant computation times. One possible mitigating strategy for this is the use of parallelization via graphics processing units (GPU). For instance, the linear dispersive algorithm used in this paper has already seen a parallel GPU implementation in [26]. Likewise, a cursory glance at the above algorithm shows that the elemental matrices can all be independently numerically integrated and computed before being combined into their global counterparts. This exposes significant parallelization and may help alleviate some of the computational burden when executed on a GPU. Similarly, it may be possible to expose parallelism in other aspects of the algorithm or to port the nonlinear dispersive theory above to other more readily parallelizable methods, such as the Gaussian Belief Propagation [27] or Discontinuous Galerkin [28] methods. This remains an active area of research for future work.

\section{ACKNOWLEDGMENT}

The authors would like to thank the Natural Sciences and Engineering Research Council (NSERC) of Canada for their support.

\section{REFERENCES}

[1] C. Gabriel, Dielectric Properties of Biological Materials, ser. Handbook of Biological Effects of Electromagnetic Fields: Bioengineering and Biophysical Aspects of Electromagnetic Fields. Boca Raton, FL.: Taylor and Francis, 2007, vol. 1.

[2] A. M. Woodward and D. B. Kell, "On the nonlinear dielectric properties of biological systems," Bioelectrochemistry and Bioenergetics, vol. 24, pp. $83-100,1990$

[3] G. T. S. Mercier, A. Palanisami, and J. H. M. Jr., "Nonlinear dielectric spectroscopy for label-free detection of respiratory activity in whole cells," Biosensors and Bioelectronics, vol. 25, pp. 2107 - 2114, 2010.

[4] R. Boyd, Nonlinear Optics, 3rd ed. Burlington, MA, USA: Academic Press, 2008.

[5] R. M. Joseph and A. Taflove, "FDTD maxwell's equations models for nonlinear electrodynamics and optics," IEEE Transactions on Antennas and Propagation, vol. 45, no. 3, pp. 364-374, Mar. 1997.

[6] K. J. Blow and D. Wood, "Theoretical description of transient stimulated raman scattering in optical fibers," IEEE Journal of Quantum Electronics, vol. 25, no. 12, pp. 2665 - 2673, Dec. 1989.

[7] J. V. Roey, J. van der Donk, and P. E. Lagasse, "Beam-propagation method: analysis and assessment," J. Opt. Soc. Am., vol. 71, no. 7, pp. 803-810, Jul. 1981.
[8] M. F. S. Ferreira, Nonlinear Effects in Optical Fibers, 3rd ed. Hoboken, New Jersey: John Wiley \& Sons, Inc., 2011, ch. 4, pp. 63-84.

[9] R. M. Joseph and A. Taflove, "Spatial soliton deflection mechanism indicated by FD-TD maxwell's equations modeling," IEEE Photonics Technology Letters, vol. 6, no. 10, pp. 1251-1254, Oct. 1994.

[10] D. Jiao and J.-M. Jin, "Time-domain finite-element modeling of dispersive media," IEEE Microwave and Wireless Components Letters, vol. 11, no. 5, pp. 220 - 222, May 2001.

[11] F. L. Teixeira, "Time-domain finite-difference and finite-element methods for maxwell equations in complex media," IEEE Transactions on Antennas and Propagation, vol. 56, no. 8, pp. 2150-2166, Aug. 2008.

[12] A. Akbarzadeh-Sharbaf and D. D. Giannacopoulos, "A stable and efficient direct time integration of the vector wave equation in the finiteelement time-domain method for dispersive media," IEEE Transaction on Antennas and Propagation, vol. 63, no. 1, pp. 314-321, Jan. 2015.

[13] _ "Finite-element time-domain solution of the vector wave equation in doubly dispersive media using mobius transformation technique," IEEE Transactions on Antennas and Propagation, vol. 61, no. 8, pp. 4158 - 4166, Aug. 2013.

[14] S. Yan and J.-M. Jin, "Theoretical formulation of a time-domain finite element method for nonlinear magnetic problems in three dimensions," Progress in Electromagnetics Research, vol. 153, pp. 33-55, 2015.

[15] — "Analysis of nonlinear electromagnetic problems using timedomain finite element method," Radio Science Meeting (Joint with AP-S Sympsium), USNC-URSI, p. 99, July 2013.

[16] - , "Three-dimensional time-domain finite-element simulation of dielectric breakdown based on nonlinear conductivity model," IEEE Transactions on Antennas and Propagation, vol. 64, no. 7, pp. 3018 - 3026, Jul. 2016.

[17] _ "Time-domain finite element modeling of nonlinear conductivity using newton's method," 2015 IEEE International Symposium on Antennas and Propagation \& USNC/URSI National Science Meeting, July 2015.

[18] B. Zhu, H. Yang, and J. Chen, "A novel finite element time domain method for nonlinear maxwell's equations based on the parametric quadratic programming method," Microwave and Optical Technology Letters, vol. 57, no. 7, pp. 1640-1645, July 2015.

[19] A. Fisher, D. White, and G. Rodrigue, "An efficient vector finite element method for nonlinear electromagnetic modeling," Journal of Computational Physics, vol. 225, no. 2, pp. 1331-1346, Feb. 2007.

[20] J. Jin, The Finite Element Method in Electromagnetics, 2nd ed. New York, NY: Wiley-IEEE Press, 2002.

[21] M.-F. Wong, O. Picon, and V. F. Hanna, "A finite element method based on whitney forms to solve maxwell equations in the time domain," IEEE Transactions on Magnetics, vol. 31, no. 3, pp. 1618 - 1621, May 1995.

[22] A. Bossavit, "Whitney forms: A class of finite elements for threedimensional computations in electromagnetism," IEE Proceedings A Physical Science, Measurement and Instrumentation, Management and Education - Reviews, vol. 135, no. 8, pp. 493 - 500, Nov. 1988.

[23] G. Mur and A. T. de Hoop, "A finite-element method for computing three-dimensional electromagnetic fields in inhomogeneous media," IEEE Transactions on Magnetics, vol. 21, no. 6, pp. 2188 - 2191, Nov. 1985.

[24] Y. Zhu and A. C. Cangellaris, Multigrid Finite Element Methods for Electromagnetic Field Modeling. Hoboken, New Jersey: John Wiley \& Sons, Inc., 2006.

[25] S. Yan and J.-M. Jin, "Analysis of nonlinear electromagnetic problems using time-domain finite element method," Radio Science Meeting (Joint with AP-S Sympsium), USNC-URSI, p. 99, July 2013.

[26] D. S. Abraham and D. D. Giannacopoulos, "Dispersive mobius transform finite-element time-domain method on graphics processing units," IEEE Transactions on Magnetics, vol. 52, no. 3, Mar. 2016.

[27] D. Fernandez, A. Akbarzadeh-Sharbaf, and D. D. Giannacopoulos, "Solving finite-element time-domain problems with gabp," IEEE Transactions on Magnetics, vol. PP, no. 99, pp. 1 - 1, Jan. 2017.

[28] S. D. Gedney, T. Kramer, C. Luo, J. Roden, R. Crawford, B. Guernsey, J. Beggs, and J. A. Miller, "The discontinuous galerkin finite element time domain method (dgfetd)," 2008 IEEE International Symposium on Electromagnetic Compatibility, Aug. 2008. 


\begin{tabular}{|c|}
\hline \\
\\
PLACE \\
PHOTO \\
HERE \\
\end{tabular}

John Doe I Biography text here.

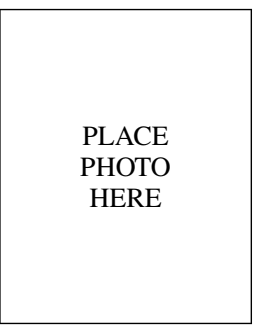

John Doe II Biography text here. 